\title{
Pulmonary Diffuse Alveolar Septal Amyloidosis
}

\author{
Yu Kurahara ${ }^{1}$, Kazunobu Tachibana ${ }^{1}$, Masanori Kitaichi ${ }^{2}$ and Seiji Hayashi ${ }^{1}$
}

Key words: amyloidosis, random distribution

(Intern Med 51: 1447-1448, 2012)

(DOI: 10.2169/internalmedicine.51.7050)

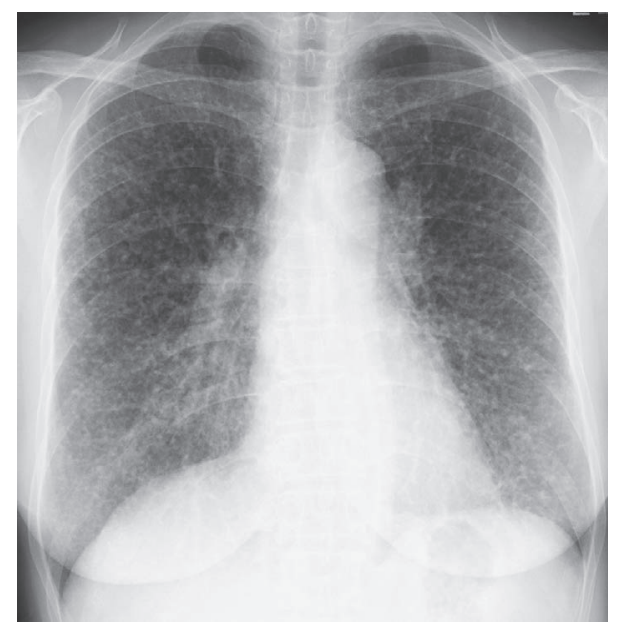

Picture 1. Chest X-ray showing bilateral small nodules.

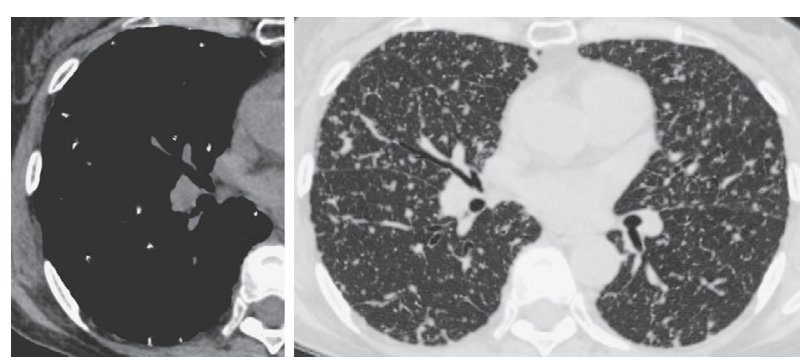

Picture 2. High-resolution computed tomography of the chest, showing interlobular septal thickening and random distribution of small nodules with calcification.

A 59-year-old woman was referred to our hospital for bilateral nodules on chest X-ray (Picture 1). High-resolution computed tomography (HRCT) showed interlobular septal thickening and random distribution of nodules with calcification (Picture 2). Transbronchial lung biopsy revealed accumulation of amyloid in the alveolar septa, the bronchiolar walls and the walls of blood vessels, which were confirmed
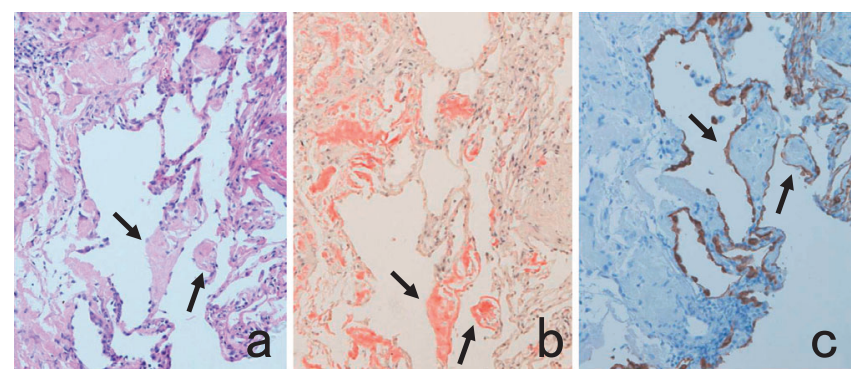

Picture 3. Histopathologic findings of the transbronchial biopsy specimens from the right lung: (a) Alveolar septa were thickened with acellular eosinophilc homogeneous materials (arrows) (Hematoxylin and Eosin staining, original magnification $\times 200$ ). (b) Multifocal thickening of alveolar septa with Congo red positive materials were observed (arrows). Congo red positive materials also deposited in the bronchiolar wall and adjacent alveolar region (Congo red stain, original magnification $\times 200)$. (c) The amyloid materials were covered on both sides with keratin-positive alveolar lining epithelial cells. Thus, amyloid depositions were confirmed in the alveolar septa in these places (arrows) (AE1/AE3 immunostain, original magnification $\times 200)$.

by Congo red stain (Picture 3). These HRCT and pathologic findings were compatible with pulmonary diffuse alveolar septal amyloidosis, and thorough investigations revealed underlying multiple myeloma (1).

Pulmonary diffuse alveolar septal amyloidosis is rare but clinically significant since it is associated with underlying disorders $(1,2)$. The HRCT patterns may be misdiagnosed as sarcoidosis, miliary tuberculosis, or metastatic carcinoma (2). Thus, we stress that randomly distributed nodules can be seen on HRCT of patients with amyloidosis, and calcification of the nodules, if any, is helpful information for differentiation.

The authors state that they have no Conflict of Interest (COI).

${ }^{1}$ Department of Internal Medicine, National Hospital Organization Kinki-Chuo Chest Medical Center, Japan and ${ }^{2}$ Department of Pathology, National Hospital Organization Kinki-Chuo Chest Medical Center, Japan

Received for publication December 7, 2011; Accepted for publication February 15, 2012

Correspondence to Dr. Seiji Hayashi, shayashi@kch.hosp.go.jp 
Intern Med 51: 1447-1448, 2012 DOI: 10.2169/internalmedicine.51.7050

\section{References}

1. Gilmore JD, Hawkins PN. Amyloidosis and the respiratory tract. Thorax 54: 444-451, 1999.
2. Urban BA, Fishman EK, Goldman SM, et al. CT evaluation of amyloidosis: spectrum of disease. Radiographics 13: 1295-1308, 1993.

(C) 2012 The Japanese Society of Internal Medicine http://www.naika.or.jp/imindex.html 